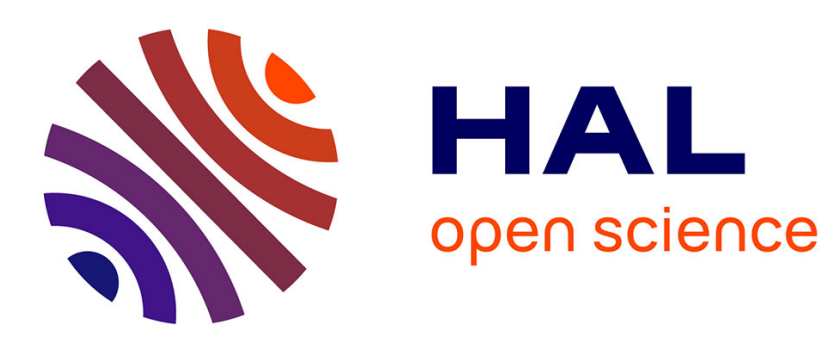

\title{
Phase Properties of Laser High-Order Harmonics Generated on Plasma Mirrors
}

F Quéré, Cédric Thaury, J-P Geindre, G Bonnaud, P Monot, Ph Martin

\section{To cite this version:}

F Quéré, Cédric Thaury, J-P Geindre, G Bonnaud, P Monot, et al.. Phase Properties of Laser High-Order Harmonics Generated on Plasma Mirrors. Physical Review Letters, 2007, 100, pp.095004. 10.1103/PhysRevLett.100.095004 . hal-01166816

\section{HAL Id: hal-01166816 https://hal.science/hal-01166816}

Submitted on 23 Jun 2015

HAL is a multi-disciplinary open access archive for the deposit and dissemination of scientific research documents, whether they are published or not. The documents may come from teaching and research institutions in France or abroad, or from public or private research centers.
L'archive ouverte pluridisciplinaire $\mathbf{H A L}$, est destinée au dépôt et à la diffusion de documents scientifiques de niveau recherche, publiés ou non, émanant des établissements d'enseignement et de recherche français ou étrangers, des laboratoires publics ou privés. 


\title{
Phase Properties of Laser High-Order Harmonics Generated on Plasma Mirrors
}

\author{
F. Quéré, ${ }^{1}$ C. Thaury, ${ }^{1}$ J-P. Geindre, ${ }^{2}$ G. Bonnaud, ${ }^{1}$ P. Monot, ${ }^{1}$ and Ph. Martin ${ }^{1}$ \\ ${ }^{1}$ Service des Photons, Atomes et Molécules, Commissariat à l'Energie Atomique, DSM/IRAMIS, CEN Saclay, \\ 91191 Gif sur Yvette, France \\ ${ }^{2}$ Laboratoire pour l'utilisation des lasers intenses, UMR 7605-CNRS-CEA-Ecole Polytechnique-Université Paris VI, \\ F91128 Palaiseau France \\ (Received 13 September 2007; published 6 March 2008)
}

\begin{abstract}
As a high-intensity laser-pulse reflects on a plasma mirror, high-order harmonics of the incident frequency can be generated in the reflected beam. We present a numerical study of the phase properties of these individual harmonics, and demonstrate experimentally that they can be coherently controlled through the phase of the driving laser field. The harmonic intrinsic phase, resulting from the generation process, is directly related to the coherent sub-laser-cycle dynamics of plasma electrons, and thus constitutes a new experimental probe of these dynamics.
\end{abstract}

PACS numbers: 52.38. $-\mathrm{r}, 42.65 . \mathrm{Ky}$, 52.59. Ye, 52.65.Rr

The generation of high-order harmonics of intense femtosecond (fs) laser pulses is one of the promising paths for the production of energetic, short-wavelength, ultrashort light pulses [1,2]. The practical characteristics of such an extreme ultraviolet (XUV) light source, e.g., divergence, duration, coherence- are largely determined by the phase properties of the harmonics, which should therefore be carefully studied in order to understand and optimize these sources. From a fundamental point of view, laser harmonics generated by driving a system with a strong laser field constitute a powerful diagnostic to investigate the dynamics of this system, and in this respect too, phase properties are of prime importance.

For these reasons, phase properties of high-order harmonics generated from atoms or molecules have been extensively studied over the past 15 years [1]. In this case, the relative phases of the harmonics are directly related to the sub-laser-cycle dynamics of the continuum electron wave-packet responsible for the generation [3]. These phases are well defined and relatively weak: harmonics are thus synchronized over a broad frequency range, and their superposition produces trains of attosecond pulses. Because these dynamics depend on laser intensity, the temporal and spatial intensity envelopes of the driving laser-pulse lead to variations of the attosecond pulses properties both in time and space [4]. These variations result in nontrivial temporal and spatial intrinsic phases of individual harmonics, which in turn affect the properties of the harmonic beam.

Reflection of a laser beam on a plasma-mirror (PM) at high intensities $\left(I \lambda^{2} \gtrsim 10^{16} \mathrm{~W} \mathrm{~cm}^{-2} \mu \mathrm{m}^{2}\right.$, where $\lambda$ is the laser wavelength and $I$ the laser intensity) provides another means for high-order harmonic generation (HHG), and is predicted to lead to attosecond XUV pulses with shorter wavelengths and higher energies than those presently generated in gases [2,5]. This Letter presents the first experimental and theoretical study of the intrinsic spatial and temporal-phase properties of individual harmonics gener- ated from plasma mirrors, for the two HHG mechanisms identified so far $[2,6,7]$, coherent wake emission (CWE) $[8,9]$ and the relativistic oscillating mirror process (ROM) $[10,11]$.

Although they correspond to very different physical processes, CWE and ROM have a common root, the Brunel mechanism [12,13], where electrons at the plasma surface are first accelerated in vacuum by the laser field, and then pushed back toward the plasma. ROM occurs as these electrons are pulled out of the plasma. For laser intensities such that $I \lambda^{2} \geqslant 1.37 \times 10^{18} \mathrm{~W} \mathrm{~cm}^{-2} \mu \mathrm{m}^{2}$, these electrons reach relativistic velocities that lead to a Doppler upshift of the reflected light wave, resulting in the production of attosecond pulses predicted to be almost Fourier-transform limited [5,14]. When Brunel electrons return to the plasma, some of them can bunch temporally. As they travel across the density gradient at the plasma surface, these electrons trigger CWE, by exciting in their wake high-frequency plasma oscillations which subsequently emit light through linear mode conversion, in the form of slightly chirped attosecond pulses [9]. CWE and ROM have recently been discriminated experimentally [6,7], through different intensity dependences, spectral ranges and spectral widths of the associated harmonics [6]. The present study extends this work by revealing extremely different phase properties of the individual harmonics associated to these two processes.

We start with a numerical study of the phase properties, and concentrate on the temporal phases $\phi_{n}(t)$ of the individual harmonics $-n$ being the harmonic order. Nontrivial phases of the individual harmonics are associated to deviations from perfect periodicity in the train of attosecond pulses [4]. Such deviations can result from the intensity envelope $I(t)$ of the laser pulse. For instance, this timedependent intensity can lead to variations of the carrierenvelope relative phase (CEP) of attosecond pulses in the train, or to a variable time-spacing between these pulses. The contribution of the first effect to $\phi_{n}(t)$ is independent 
of harmonic order, while the contribution of the second is proportional to $n$. A temporal dependence of the target response can also affect the periodicity of the train, and lead to additional contributions to the phases of the harmonics. In the case of PM's, the density gradient at the plasma-vacuum interface evolves during the laser pulse because of ion motion, and this gradient influences both CWE and ROM [7].

To quantify these different effects, we performed 2D particle-in-cell (PIC) simulations using the relativistic kinetic code CALDER [15]. The reflected electromagnetic field in vacuum is frequency filtered around a group of harmonics, to obtain the train of attosecond pulses generated upon reflection on the plasma. Figures 1(a)-1(c) show, for different cases, the temporal positions of the attosecond pulses maxima within each optical cycle, $t_{e}(k)=t_{p}-k$, where $t_{p}$ is absolute time of a maximum (in units of the laser optical period $T_{L}$ ) and $k$ the number of the corresponding laser optical cycle. The time origin is chosen such that zeros of the laser field occur at $t_{e}=0$. Hollow dots correspond to cases where the normalized vector potential $a_{0}=e E_{0} / \omega_{0} m_{e} c$ at the peak of the pulse is 0.2 , and CWE dominates over the entire pulse. Full triangles correspond to $a_{0}=2$, and we have selected a spectral range where harmonics are only due to ROM (i.e., harmonics beyond the maximum plasma frequency [6]).

To separate the effect of the laser intensity envelope from that of the time-varying plasma conditions, we first performed simulations with fixed ions [Fig. 1(a)]. In the

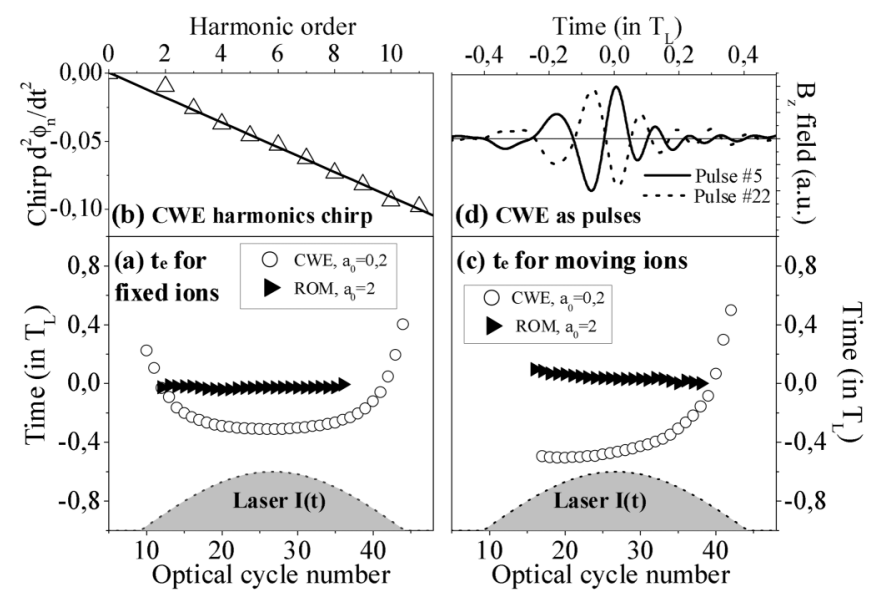

FIG. 1. Emission times of CWE and ROM attosecond pulses (superposition of harmonics 4 to 10 for $\mathrm{CWE}$, and 11 to 35 for ROM), for a plasma of maximum density $110 n_{c}\left(n_{c}\right.$ critical density at the laser frequency), driven by a 35-cycle $\cos ^{2}(t)$ laser pulse at $45^{\circ}$ incidence. In (a), ions are kept fixed, and the exponential density gradient scale length is $\lambda / 100(\lambda / 30)$ for CWE (ROM). (b) The chirp parameter of individual CWE harmonics obtained from PIC simulations in this case. In (c) ions are allowed to move, and the initial density gradient is infinitely steep. (d) The fields of two individual CWE attosecond pulses in the train in this case. case of CWE, $t_{e}$ then varies by about $0.7 T_{L}$ over the entire laser pulse. The time spacing between successive attosecond pulses, $t_{e}(k+1)-t_{e}(k)$, increases during the laser pulse, leading to a negative chirp (i.e., from blue to red) of the individual harmonics. In addition, we observe that neither the CEP phase nor the chirp of the attosecond pulses vary significantly along the train. The variable time spacing is thus the dominant contribution to the chirp, leading to a linear variation of the chirp parameter $\partial^{2} \phi_{n} / \partial t^{2}$ as a function of the harmonic order $n$ [Fig. 1(b)] [4]. In the case of ROM, less attosecond pulses are emitted, because of a stronger dependence of its efficiency on intensity compared to CWE [6]. In addition, $t_{e}$ hardly varies along the laser pulse: in strong contrast with CWE, this mechanism leads to an almost perfect periodic time of emission [16]. Since the CEP of the attosecond pulses is also observed to be constant along the train, individual ROM harmonics have a negligible temporal chirp.

To study the additional effect of the temporal evolution of the plasma conditions, we have performed simulations with mobile ions, with initial ion and electron temperatures of, respectively, 0.1 and $0.5 \mathrm{keV}$ [Fig. 1(c)]. The emission time $t_{e}$ is now observed to vary in the case of ROM, but this variation is small -less than $T_{L} / 10$ over the entire laser pulse. More striking effects occur for CWE. CWE attosecond pulses now start to be emitted later in the 35-cycle long $(\approx 90 \mathrm{fs}$ at $800 \mathrm{~nm}$ ) laser pulse, leading to the suppression of the first part of the emission time curve $t_{e}(k)$. This is because CWE requires a short, but nonvanishing density gradient, to allow for light emission by plasma oscillations $[9,17]$. This density gradient develops during the laser pulse because of plasma expansion, and reaches a scale length of about $\lambda / 70$ at the end of the laser pulse. In the second half of the pulse, $t_{e}$ follows a curve similar to what is obtained with fixed ions. In addition to this effect, a drift of the attosecond pulses CEP now occurs between the beginning and the end of the train, with a total amplitude of $\approx \pi$ in the present case [Fig. 1(d)].

If the same simulation of CWE-i.e. with moving ions - is now performed with a constant laser intensity, $t_{e}$ is observed to be almost constant, but the same drift of the CEP still occurs. This leads to two conclusions. (i) Variations of the CEP are induced by the temporal dependence of the gradient scale length $L$. This is consistent with the CWE model, where the field of the emitted attosecond pulses depends on the temporal evolution of the plasma oscillations in the density gradient, which is in turn largely determined by the spatial profile of this gradient [9]. (ii) Variations of $t_{e}$ are mostly due to the temporal evolution of the laser intensity, as in the case of fixed ions, but now with an additional temporal drift due to the influence of the gradient length on this emission time. Comparing these different simulations, we find that, in the experimentally relevant case of moving ions and time-dependent laser intensity, the variation of the emission time is the dominant contribution to the intrinsic phase of CWE har- 
monics, exceeding that of the CEP variations by a factor of more than 10 .

We now turn to experiment to test some of these predictions. We first consider the temporal-phase properties. A nonvanishing and nontrivial (i.e., not linear) temporal phase $\phi_{n}(t)$ of harmonic $n$ leads, in the spectral domain, to a broadening of the harmonic peak, compared to the case of an harmonic pulse of same duration with no temporal phase. If a phase $\phi_{L}(t)$ is also applied to the driving laser pulse, it combines with the intrinsic phase $\phi_{n}^{i}$ induced during the HHG process, and leads to a total phase $\phi_{n}(t)=$ $\phi_{n}^{i}(t)+n \phi_{L}(t)$. The magnitude of $\phi_{n}(t)$, and hence the spectral width of the harmonics, can thus be modified either enhanced, or reduced if $\phi_{n}^{i}(t) \neq 0$ - by using an adequate temporal phase of the laser pulse [18].

Figure 2(a) shows the result of such a measurement for CWE harmonics generated on a silica target, using the UHI10 laser system at Saclay Laser Interaction Center (SLIC), a 10-TW 60-fs 790-nm titanium:sapphire laser system, with a temporal contrast of $10^{-10}$ obtained with a double plasma-mirror optical switch $[6,19]$. The temporal phase of the driving laser pulse was changed by applying a chirp, simply moving one of the gratings in the compressor. The spectral width $\Delta \omega$ of harmonic 13 was measured using an imaging flat-field spectrometer, consisting in a toroidal mirror followed by a flat varied line spacing grating, with a resolution better than $10^{-2} \omega_{0}\left(\omega_{0}\right.$ being the laser frequency) in this spectral range. It is plotted as a function of the dimensionless chirp parameter $\xi$ of the laser pulse, related to the pulse duration $\tau$ by $\tau=\tau_{\mathrm{FTL}} \sqrt{1+\xi^{2}}$, where $\tau_{\text {FTL }}$ is the Fourier-transform-limited (FTL) pulse duration.
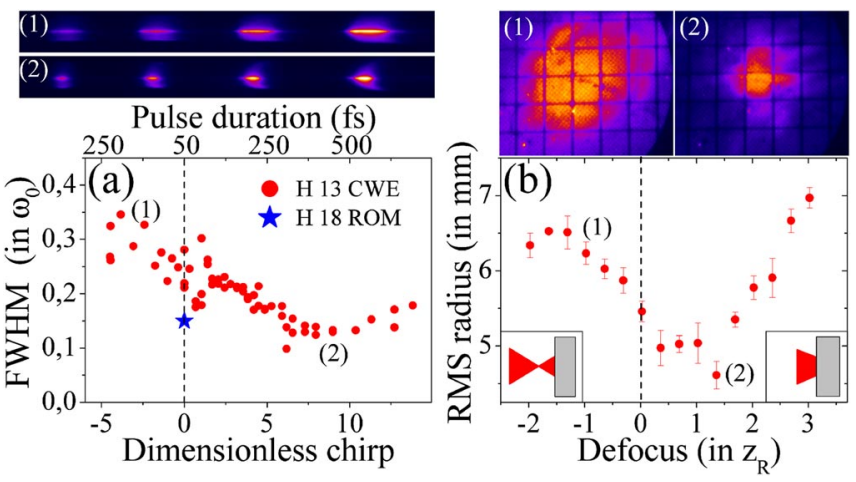

FIG. 2 (color online). (a) Measured spectral width of harmonic 13 generated by CWE ( $a_{0}=0.25$ for FTL pulses), as a function of the dimensionless chirp parameter $\xi$ of the laser pulse. The images show HHG spectra obtained around harmonic 13 for the two different chirps indicated on the graph. The spectral width of harmonic 18 generated by ROM $\left(a_{0}=2\right)$ with a FTL laser pulse is shown for comparison. (b) Diameter of the CWE harmonic beam in the far field, generated on a plastic target (orders $\approx 13$ to 15 filtered using a $100 \mathrm{~nm}$ thick Al filter), as a function of the distance between target surface and best focus $\left(a_{0}=0.6\right.$ at best focus). The two upper images show the beam patterns for two different positions.
As predicted by our theoretical study, $\Delta \omega$ reaches a minimum when the laser pulse is positively chirped $(\xi>0)$, i.e., when its phase compensates, at least partially, for the negative intrinsic phase of the CWE harmonic.

The spectral width of the 18th ROM harmonic [6] generated on a plastic target by a FTL 60 fs pulse from UHI10 is also shown on this graph. It is comparable to the smallest spectral width obtained for CWE harmonics with a positively chirped laser pulse, although ROM harmonics are emitted during a shorter fraction of the laser pulse (Fig. 1). Following our numerical study, this observation is explained by the fact that the intrinsic phase of ROM harmonics is smaller than that of CWE.

These temporal effects have an exact analogue in the spatial domain. In CWE, the spatial dependence of the laser intensity at the focus results in a time of emission of the attosecond pulses that varies spatially, and thus in an intrinsic spatial phase $\psi_{n}(r)$ of the harmonics. This phase increases the divergence of the harmonic beam. In analogy to the experiment of Fig. 2(a), it can be partially compensated by a spatial phase $\psi_{L}(r)$ of the laser beam of opposite sign, thus leading to a reduced divergence of the harmonic beam. $\psi_{L}(r)$ can be controlled by moving the target surface away from the best focus of the beam, to a point where the beam wave front has a nonvanishing curvature.

Figure 2(b) shows the root-mean square radius of a CWE harmonic beam $37 \mathrm{~cm}$ away from the target, as a function of the distance between the target surface and the laser best focus. The minimum divergence of the harmonic beam is obtained when the best focus of the laser beam is placed beyond the target surface: in this situation, the phase advance of the laser field at the edges of the beam compensates for the delayed emission time of the attosecond pulses due to the lower laser intensity (Fig. 1). All these result strongly support the numerical findings presented above, and demonstrate the coherent control of XUV beams emitted by overdense plasmas.

We now analyze the physics of HHG from PM's to interpret these results. Because it is based on a Doppler effect, ROM is expected to generate short-wavelength light most efficiently when outgoing Brunel electrons reach their maximum velocity along the direction of the reflected beam. In contrast, CWE is triggered when Brunel electrons travel across the overdense part of the plasma. These different stages of the Brunel mechanism correspond to well-defined areas of the $x-p_{x}$ phase-space density (where $x$ is the spatial coordinate along the target normal) of the plasma electrons at specific times [see white dashed boxes in Figs. 3(a) and 3(b)]. We have therefore used the $1 D \frac{1}{2}$ relativistic kinetic EUTERPE code [20], in the boosted frame [21], to select the macroparticles passing through these areas at these times. The trajectories along $x$ of the filtered macroparticles corresponding to Brunel electrons involved in ROM and CWE are, respectively, shown in Figs. 3(c) and 3(e), for two laser intensities in each case. 

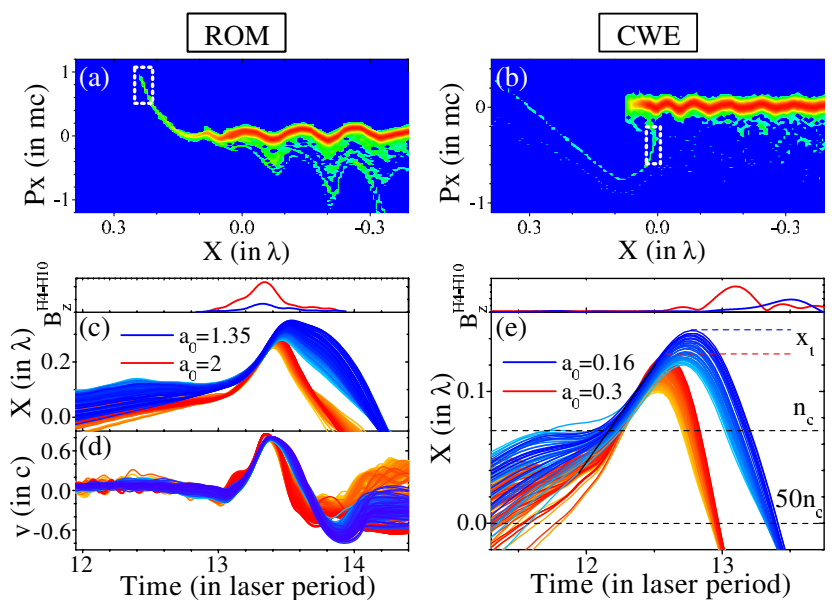

FIG. 3 (color online). (a)-(b) $x-p_{x}$ phase-space densities of plasma electrons at two different times, corresponding, respectively, to (a) the frequency upshift of the reflected light by relativistic outgoing Brunel electrons, and (b) to the penetration of Brunel electrons in the density gradient. In the lower graphs, electrons occupying the areas in the white dashed boxes have been selected. (c) Trajectories and (d) velocity $v$ (along specular direction) of Brunel electrons responsible for ROM harmonics (fixed ions with imposed gradient of $L=\lambda / 30$ ). (e) Trajectories of Brunel electrons triggering plasma oscillations (same conditions with $L=\lambda / 60$ ). In each case, two sets of trajectories are shown, corresponding to two laser intensities. The upper panels show the amplitude of the magnetic field $B$ of the corresponding attosecond pulses.

As far as ROM is concerned, outgoing Brunel electrons always reach their maximum velocity at the same instant of the laser optical cycle [Fig. 3(d)], whatever the laser intensity. This is why the emission time of ROM attosecond pulses does not vary with intensity [upper panel in Figs. 3(c) and 3(d)]. In contrast, in CWE, the instant where Brunel electrons reach the dense part of the plasma depends on the laser intensity [Fig. 3(e)]. The time where plasma oscillations are triggered therefore also depends on intensity, and so does the emission time of the CWE attosecond pulse they emit [upper panel in Fig. 3(e)].

In $[6,9]$, we suggested that the variable delay in the emission time of CWE attosecond pulses could be accumulated once Brunel electrons propagate in the overdense part of the plasma, with a velocity that depends on laser intensity [22]. In Fig. 3(e), this effect only accounts for about $40 \%$ of the final delay. The other contribution to the delay comes from the fact that the turning point $x_{t}$ of Brunel electrons [Fig. 3(e)] goes further toward vacuum as intensity goes down. Because of this larger excursion, Brunel electrons return later to the dense part of the plasma for decreasing intensities. PIC simulations show that the relative contribution of these two effects depends on the gradient scale length $L$, the effect of the varying excursion becoming dominant for $L \lesssim \lambda / 60$.
From this study, it clearly appears that high-order harmonics from PMs provide a unique wealth of information on the dynamics of the laser-plasma interaction, partly encoded in their phase properties. These phase properties, and hence the characteristics of the associated XUV light beam, can be coherently controlled through the phase of the driving laser field. As far as applications are concerned, this work shows that ROM in the moderately relativistic interaction regime present several advantages over CWE: in addition to a greater spectral extension, the associated harmonics should have a smaller divergence and a better transverse coherence, due to their negligible intrinsic phase.

We gratefully acknowledge E. Lefebvre for providing the code CALDER and for his assistance on using this code, as well as P. d'Oliveira, F. Réau, and M. Bougeard for their precious technical support.

[1] P. Agostini and L. DiMauro, Rep. Prog. Phys. 67, 813 (2004).

[2] B. Dromey et al., Nature Phys. 2, 456 (2006); Phys. Rev. Lett. 99, 085001 (2007)

[3] Y. Mairesse et al., Science 302, 1540 (2003).

[4] K. Varju et al., J. Mod. Opt. 52, 379 (2005); Phys. Rev. Lett. 95, 243901 (2005).

[5] L. Plaja, L. Roso, K. Rzazewski, and M. Lewenstein, J. Opt. Soc. Am. B 15, 1904 (1998).

[6] C. Thaury et al., Nature Phys. 3, 424 (2007).

[7] A. Tarasevitch, K. Lobov, C. Wunsche, and D. von der Linde, Phys. Rev. Lett. 98, 103902 (2007).

[8] U. Teubner et al., Phys. Rev. Lett. 92, 185001 (2004).

[9] F. Quéré et al., Phys. Rev. Lett. 96, 125004 (2006).

[10] S. V. Bulanov et al., Phys. Plasmas 1, 745 (1994).

[11] R. Lichters and J. Meyer-ter-Vehn, and A. Pukhov, Phys. Plasmas 3, 3425 (1996).

[12] F. Brunel, Phys. Rev. Lett. 59, 52 (1987).

[13] Because plasma mirrors have extremely sharp density gradients, resonance absorption does not occur.

[14] S. Gordienko, A. Pukhov, O. Shorokhov, and T. Baeva, Phys. Rev. Lett. 93, 115002 (2004).

[15] E. Lefebvre et al., Nucl. Fusion 43, 629 (2003).

[16] Simulations show that, in the ultrarelativistic regime, the emission time of ROM attosecond pulses depends on intensity. D. an der Brügge and A. Pukhov, Phys. Plasmas 14, 093104 (2007).

[17] This excludes coherent transition radiation as the emission mechanism, because this process would also occur for a steplike density gradient.

[18] Z. Chang et al., Phys. Rev. A 58, R30 (1998).

[19] A. Lévy et al., Opt. Lett. 32, 310 (2007).

[20] G. Bonnaud and C. Reisse, Nucl. Fusion 26, 633 (1986).

[21] P. Gibbon and A. R. Bell, Phys. Rev. Lett. 68, 1535 (1992).

[22] A similar effect for harmonics emitted from the rear side of thin foils is discussed on p. 7 of K. Eidmann et al., Phys. Rev. E 72, 036413 (2005). 